\title{
Meillassoux y Deleuze en torno a la inmanencia
}

\author{
Meillassoux and Deleuze on Immanence
}

Marcelo Antonelli*

Resumen: Quentin Meillassoux (1967) es un filósofo francés que ha cobrado notoriedad a partir de la publicación de Après la finitude. Essai sur la nécessité de la contingence en 2006. Su obra se inscribe en el Realismo Especulativo, movimiento de reciente expansión en el campo filosófico contemporáneo que promueve una ontología realista. Además de reivindicar una filosofía materialista, Meillassoux defiende la inmanencia ya en su tesis doctoral L'inexistence divine y, especialmente, en el artículo "L'immanence: d'outre-monde" (2009), donde afirma que una verdadera filosofía de la inmanencia no puede fundamentarse en un pensamiento de la finitud sino en una ética de la inmortalidad. Meillassoux opone su perspectiva, aparentemente paradójica, de una inmanencia "de otro mundo" a los pensamientos contemporáneos de la inmanencia, esencialmente al de Deleuze, quien hizo de dicho concepto un blasón de su proyecto filosófico. En este artículo queremos ensayar una confrontación entre la idea de Meillassoux de una creencia en el Mundo de la Justicia y la propuesta deleuziana de una creencia en este mundo con vistas a elucidar convergencias y divergencias relevantes en sus abordajes de la inmanencia y sus concepciones del vínculo entre la filosofía y el ateísmo.

Palabras clave: creencia, inmortalidad, finitud, trascendencia, moral

Abstract: French Philosopher Quentin Meillassoux (1967) has become renowned since the publication of Après la finitude. Essai sur la nécessité de la contingence in 2006. His work is set within Speculative Realism, a vibrant recent movement in the upsurge,

* Doctor en Filosofía (Universidad de Buenos Aires-Paris 8). Investigador Asistente en el Consejo Nacional de Investigaciones Científicas y Técnicas (CONICET) y Profesor Adjunto Regular en la Universidad Pedagógica Nacional y en la Universidad Nacional de San Martín. Es Investigador Principal en el Centro de Investigaciones Filosóficas (Buenos Aires). Su área de investigación es la filosofía francesa de la segunda mitad del siglo XX (en particular, la obra de Gilles Deleuze), la corriente biopolítica (Foucault, Agamben, Negri, Esposito) y los nuevos realismos en la filosofía continental (Meillassoux, DeLanda, Harman, Ferraris). Dirección electrónica: antonelli.ms@gmail.com 
which enhances a realistic ontology. Beyond supporting a materialistic philosophy, Meillassoux has embraced immanence from the start of his career; this is clearly depicted in his $\mathrm{PhD}$ thesis L'inexistence divine. He especially fostered it further in his article "L'immanence: d'outre-monde" (2009), in which he states that a true philosophy on immanence cannot be based on the thought of finitude, but on the ethics of immortality. Meillassoux contrasts his perspective, apparently paradoxical, of "otherwordly immanence" to contemporary thoughts on immanence, principally Deleuze's, who made of this concept the scaffolding of his philosophical project. In this article we aim at portraying a confrontation between Meillassoux's belief in the World of Justice and Deleuze's proposal of a belief in this world. This shall enable us to shed light on relevant similarities and differences regarding their approaches to immanence and their conception of the bond between philosophy and religion.

Key words: belief, immortality, finitude, transcendence, moral

\section{Meillassoux, Deleuze, la inmanencia}

1. Quentin Meillassoux es un filósofo francés nacido en París en 1967, hijo del antropólogo Claude Meillassoux, que ha cobrado notoriedad a partir de la aparición de Après la finitude. Essai sur la nécessité de la contingence en 2006. Publicó un libro sobre Mallarmé (Le Nombre et la sirène. Un déchiffrage du Coup de dés de Mallarmé, 2011) y otro sobre metafísica y ciencia ficción (Métaphysique et fiction des mondes hors-science, 2013), así como numerosos artículos y capítulos de libros. Su tesis doctoral L'Inexistence divine, defendida en 1997 y aún no publicada, ${ }^{1}$ anticipa en buena medida los temas de sus trabajos posteriores.

Après la finitude pasó más bien desapercibido en el mundo filosófico francés, no obstante lo cual se tradujo rápidamente al inglés y tuvo un impacto significativo en el ámbito anglosajón. ${ }^{2}$ De Meillassoux se ha dicho que es "la estrella ascendente de la filosofía francesa"3 y Peter Hallward lo considera el filósofo francés que se destacó más rápidamente en el mundo anglófono

\footnotetext{
1 Según Harman, la versión ampliada de la tesis será publicada, aún sin fecha definitiva, en 3 volúmenes. Cfr. Harman, G., Quentin Meillassoux. Philosophy in the making, Edinburgh, EUP, 2011, p. 91.

${ }^{2}$ Cfr. Ibidem, p. 159.

3 Duru, M., "La résurrection qui vient...", Philosophie Magazine, 25, 2012-2013 [http://www.philomag.com/les-idees/la-resurrection-qui-vient-6491].
} 
desde Derrida en los años $60 ;{ }^{4}$ además, ha recibido comentarios favorables de Zizek $^{5}$ y, en especial, de Badiou, que es uno de sus referentes más importantes. ${ }^{6}$

Meillassoux ha sido asociado al Realismo o Materialismo Especulativo, ${ }^{7}$ movimiento integrado por autores de su generación (Graham Harman, Iain Hamilton Grant, Ray Brassier) que suelen ser agrupados como parte de un "giro especulativo" en filosofía. ${ }^{8}$ Es posible constatar que confluyen, junto con el Nuevo Realismo (Maurizio Ferraris, Markus Gabriel) y el Realismo Virtual (DeLanda), en una visión crítica de la posmodernidad y del rumbo filosófico anti-realista del siglo XX, frente al cual proponen, con variantes y matices, una vuelta a la ontología realista, esto es: a la independencia y autonomía de la realidad, tanto en sus aspectos observables como en los inobservables, respecto de la mente humana. ${ }^{9}$

${ }^{4}$ Cfr. Harman, G., Quentin Meillassoux. Philosophy in the making, p. 159.

${ }^{5}$ No obstante, Zizek señala sus desacuerdos con Meillassoux (entre otros, el uso del infinito no totalizable de Cantor). Cfr. Zizek, S. y Woodard, B., "Interview", en Bryant, L., Srnicek, N. y Harman, G. (eds.), The Speculative Turn. Continental materialism and realism, Melbourne, Re.press, 2011.

${ }^{6}$ Así lo explicita el propio autor en Meillassoux, Q., Après la finitude. Essai sur la nécessité de la contingence, Paris, Du Seuil, 2006, p. 141.

7 Meillassoux emplea el término "realismo" como sinónimo de "materialismo" en Meillassoux, Q., "Presentation”, en Mackay, R. (ed.), Collapse III, Falmouth, Urbanomic, 2007, p. 426. Anna Longo, sin embargo, argumenta que es más acertado denominar a su proyecto filosófico "Materialismo" antes que "Realismo". Cfr. Longo, A., "The contingent emergence of thought: a comparison between Meillassoux and Deleuze", en Meillassoux, Q., Time without becoming, ed. por A. Longo, Mimesis International, 2014, pp. 33-35.

8 Para un panorama general de los principales autores, temas y problemas del Realismo Especulativo, véanse Bryant, L., Srnicek, N. y Harman, G. "Towards a Speculative Philosophy”, en Bryant, L., Srnicek, N. y Harman, G. (ed.): The Speculative Turn, pp. 1-18.; Gratton, P., Speculative realism. Problems and prospects, London/New York, Bloomsbury, 2014; Van der Tuin, E. y Dolphjin, R. (eds.), New Materialism: Interviews and Cartographies, Michigan, Open Humanities Press, 2012; Delanda, M. y Harman, G., The rise of realism, Cambridge, Polity Press, 2017.

9 Cfr. Delanda, M., Intensive science and virtual philosophy, London/New York: Bloomsbury, 2002, p. XII. 
2. Gratton y Ennis sostienen que Deleuze es una "fuerte influencia" en Meillassoux dado que éste despliega nuevas versiones de la inmanencia y la virtualidad. ${ }^{10}$ A nuestro juicio, esta apreciación exagera la importancia que tiene el primero en el segundo. Si bien comparten el interés por ciertos temas y conceptos, sus enfoques son heterogéneos, como pondremos de manifiesto aquí respecto de la inmanencia. ${ }^{11}$

Además, con la excepción de "Substraction and contraction: Deleuze, immanence, and Matter and Memory", las referencias a Deleuze que encontramos en la obra de Meillassoux son breves y dispersas. ${ }^{12}$ Así, en Après la finitude opina que Deleuze es "un subjetivista metafísico que absolutizó un conjunto de rasgos de la subjetividad, hipostasiada como Vida (o "una vida")". ${ }^{13}$ En diálogo con Harman, afirma que Deleuze fue "el más grande bergsoniano del siglo XX", especialmente en sus concepciones de lo virtual y la intensidad; Deleuze habría sido, junto con Badiou, el último gran metafísico

${ }^{10}$ Cfr. Gratton, P. y Ennis, J. (eds.), The Meillassoux Dictionary, Edinburgh, EUP, 2015, p. 3.

11 Acerca de la virtualidad, Meillassoux sostiene que la distinción entre potencialidad y virtualidad es gnoseológica antes que ontológica, al contrario de lo que ocurre en Deleuze. Cfr. Meillassoux, Q., "Potentiality and Virtuality", en Bryant, L., Srnicek, N. y Harman, G. (ed.), The Speculative Turn, p. 232. En otra línea de indagación, Anna Longo coteja a Meillassoux con Deleuze respecto de la emergencia del pensamiento. Cfr. Longo, A., "The contingent emergence of thought: a comparison between Meillassoux and Deleuze", en Meillassoux, Q., Time without becoming, pp. 31-50.

${ }^{12}$ Meillassoux, Q., "Substraction and contraction: Deleuze, immanence, and Matter and Memory", en Mackay, R. (ed.), Collapse III, pp. 63-107. Badiou había interpretado a Deleuze como un pre-socrático en Badiou, A., Delenze: La clameur de l'être, Paris, Hachette, 1997, p. 150.

${ }^{13}$ Cfr. Meillassoux, Q., Après la finitude, p. 51; también Meillassoux, Q., " «There is contingent being independent of us, and this contingent being has no reason to be of a subjective nature » : Interview with Quentin Meillassoux", en Van Der Tuin, I. y Dolphjin, R. (eds.), New Materialism: Interviens and Cartographies, pp. 71-81. A nuestro juicio, adjudicar a Deleuze un "subjetivismo vitalista" es desacertado porque su vitalismo, anclado en la vida en tanto línea abstracta, tiende más bien a la dessubjetivación. Cfr. Antonelli, M., "Vitalismo y desubjetivación. La ética de la prudencia en Gilles Deleuze", Signos Filosóficos, UNAM, 15, 30, 2013, pp. 89-117. 
francés. ${ }^{14}$ En el artículo “L'Immanence D’Outre Monde” hay dos menciones también sucintas, vinculadas con el Eterno Retorno y la inmanencia, sobre las que nos detendremos en este escrito. ${ }^{15}$

3. Como señalamos, Meillassoux y Deleuze se han ocupado del concepto de inmanencia, que forma parte de la tradición filosófica occidental desde sus inicios y ha adquirido recientemente una indudable relevancia, al punto de volverse un criterio ordenador en el campo de la filosofía moderna. ${ }^{16}$ En el caso de Deleuze, se trata de uno de las nociones fundamentales de su filosofía. Además de su idea de plano de inmanencia, ${ }^{17}$ el capitalismo es descripto como un sistema inmanente, ${ }^{18}$ la moral basada en valores universales es sustituida por una ética anclada en valores inmanentes, ${ }^{19}$ entre otros usos que ha hecho del término. ${ }^{20}$ A nuestro juicio, el pensamiento deleuziano se caracteriza por la reivindicación irrestricta de la inmanencia y la recusación de toda trascendencia.

Respecto de Meillassoux, la noción estaba presente ya en su tesis doctoral, en la cual sostiene que no hay nada trascendente al mundo que pueda limitar su potencia creadora de novedades o que garantice la necesidad de las leyes de la naturaleza. Asevera que su filosofía es un pensamiento de la

14 Meillassoux, Q., "«There is contingent being independent of us, and this contingent being has no reason to be of a subjective nature »: Interview with Quentin Meillassoux", p. 171.

${ }^{15}$ Meillassoux, Q., “L'Immanence D’Outre Monde”, ETHICA, Rio de Janeiro, 16, 2, 2009, pp. 39-71.

${ }^{16}$ Véase Agamben, G., "L'immanence absolue", en Alliez, E. (dir.), Gilles Deleuze. Une vie philosophique, Paris, Synthélabo, 1998, p. 187; Ciccarelli, R., Immanenza. Filosofia, diritto e politica della vita dal XIX al XX secolo, Bologna, Il Mulino, 2008.

${ }_{17}$ Véase Antonelli, M., El concepto de inmanencia en la filosofía de Deleure, tesis doctoral UBA-Paris 8, defendida el 14 de marzo de 2012.

${ }^{18}$ Véase Antonelli, M., "El capitalismo según Gilles Deleuze: inmanencia y fin de la historia", Cuadernos de Filosofia, UBA, 57, 2011, pp. 51-66.

19 Véase Antonelli, M., "El concepto de inmanencia práctica en Deleuze", Ideas y Valores, Universidad Nacional de Colombia, LXVI, 164, 2017, pp. 317-341.

${ }^{20}$ Véase Antonelli, M., "Pensar la inmanencia: Gilles Deleuze y François Jullien", Eidos. Revista de filosofía de la Universidad del Norte, 19, 2013, pp. 12-45; y Antonelli, M., "Sobre el origen del concepto de inmanencia en Gilles Deleuze", INTERthesis, Revista Internacional Interdisciplinar, UFSC, 11, 2, 2014, pp. 15-36. 
inmanencia en el sentido de "una discursividad comprensible sobre el mundo en su esencia última". ${ }^{21}$ Esta veta inmanentista reapareció en "L'immanence d'outre monde", donde declara que su filosofía de la inmanencia es más coherente que la de Deleuze.

En este trabajo queremos analizar la concepción de la inmanencia que Meillassoux presenta en el mencionado artículo y evaluar sus críticas a la versión deleuziana. A tal fin, examinaremos su propuesta de una creencia en el Mundo de la Justicia y la confrontaremos con la idea deleuziana de una creencia en este mundo. Según nuestro parecer, cotejar estas perspectivas permite poner de manifiesto convergencias y divergencias filosóficamente significativas, así como enriquecer un cruce poco explorado hasta el momento. 22

Recurriremos a ciertas posiciones deleuzianas (sobre Dios, el ateísmo, la moral) a fin de agregar elementos implícitos o ausentes el planteo de Meillassoux. Si bien nos concentraremos en "L'Immanence D'Outre Monde" porque es el texto donde explicita su crítica a Deleuze, ampliaremos con

${ }^{21}$ Cfr. Meillassoux, Q., L'Inexistence divine, tesis doctoral, Université de Paris I, Département de philosophie, 1997, pp. 378-379; “Appendix: Excerpts from L'Inexistence divine", en Harman, G., Quentin Meillassoux. Philosophy in the making, pp. $175-238$.

22 Respecto del estado de la cuestión, si bien existe abundante bibliografía sobre el pensamiento deleuziano y también sobre la obra de Meillassoux, son escasos los estudios que los han abordado en conjunto. Longo y Abadi han ensayado entrecruzarlos, aunque no en torno a los temas de este artículo; Ramírez se ocupa de las críticas de Meillassoux a Deleuze, pero sin tomar distancia conceptual; Gratton, y Gratton y Ennis exponen sucintamente algunos puntos de desacuerdo. Cfr. Longo, A., "The contingent emergence of thought: a comparison between Meillassoux and Deleuze"; Abadi, D., "Some possible relations between Quentin Meillassoux's "speculative materialism» and Gilles Deleuze's "transcendental empiricism ", Journées "Les temps des choses, la substance du temps", 21 et 22 mai 2015, Université Paris 8; Ramírez, M. T., "Devenir inmortal. La crítica de Quentin Meillassoux a la filosofía de la inmanencia de Gilles Deleuze", Signos Filosóficos, XVIII, 35, enero-junio 2016, pp. 32-51 ; Gratton, P., Speculative realism. Problems and prospects; Gratton, P. y Ennis, J. (eds.), The Meillassoux Dictionary. 
referencias a otros trabajos, en especial a Après la finitude. ${ }^{23}$ Conviene aclarar que no pretendemos reconstruir el conjunto del pensamiento de Meillassoux, lo cual excedería la extensión de este escrito, sino sólo ofrecer un marco de inteligibilidad a las tesis sobre las que nos concentraremos.

\section{Pensamiento especulativo y escatología irreligiosa}

1. En “L'Immanence D’Outre Monde", Meillassoux desarrolla dos tesis que había adelantado en Après la finitude: primero, la especulación, entendida como el pensamiento del absoluto, sólo es posible si rechazamos el principio metafísico de razón suficiente; segundo, la especulación es acompañada por una postura no religiosa ni atea sino "irreligiosa", comprendida como una "escatología de la inmortalidad". La tercera tesis, que concierne puntualmente nuestro tema y que el autor no había expuesto previamente, sostiene que "una filosofía de la verdadera inmanencia no pasa por un pensamiento de la finitud sino por una ética de la inmortalidad". 24

De acuerdo con el primer postulado, Meillassoux busca reactivar una filosofía especulativa, aunque no metafísica, que acceda al absoluto, esto es, a una verdad eterna al margen de las variaciones psicológicas, históricas, lingüísticas de nuestra relación con el mundo. Afirma la contingencia radical de todas las cosas, su "irrazón", que es una propiedad que consiste en que ellas pueden sin razón volverse diferentes de como son. El "Súper-caos" (o "Híper-caos" o "Súper-contingencia") designa el caos eterno que puede hacer emerger o perecer un mundo, así como destruir las leyes presentes de la naturaleza. Meillassoux denomina "principio de factualidad" al hecho de que,

23 Algunas tesis de "L’Immanence D’Outre Monde" (el dilema espectral, la inexistencia divina, la idea de que Dios no existe todavía) habían sido publicadas en Meillassoux, Q., "Dueil à venir, dieu à venir", Paris, Ed. Ismael, 2017, reedición del artículo publicado en Critique, "Dieu", 704-705, janvier-février 2006, Paris, Minuit, pp. 105-115. [https://editions-ismael.com]. No obstante, en dicho trabajo está ausente la perspectiva sobre la inmanencia que aparece en "L'Immanence D'Outre Monde" y que constituye el objeto de nuestro estudio: la necesidad de la creencia en el mundo de la justicia, la interpretación del Eterno Retorno, la asociación entre inmanencia y ética de la inmortalidad.

${ }^{24}$ Meillassoux, Q., “L'Immanence D’Outre Monde”, pp. 39-40. 
siendo todas las cosas contingentes, lo único que no podemos relativizar es la contingencia misma -que es, por tanto, necesaria. ${ }^{25}$

Esta perspectiva había sido presentada en Après la finitude a propósito del problema de la "ancestralidad", esto es, la producción de enunciados científicos sobre realidades anteriores a toda relación del hombre con el mundo. ${ }^{26}$ Pensar la ancestralidad equivale a pensar un mundo sin pensamiento, lo cual obliga a romper con el dictum moderno acerca de que ser es "ser un correlato". El correlacionismo sostiene el carácter insuperable de la correlación entre el ser y el pensamiento, pero Meillassoux quiere rehabilitar la conexión del pensamiento con lo absoluto comprendido como lo desligado, lo separado del pensamiento, lo no relativo a nosotros. ${ }^{27}$

Con vistas a ello, el autor absolutiza la facticidad -i.e., el hecho de que "hay" sin razón-: en lugar de ser la experiencia que el pensamiento hace de sus límites, debemos considerarla la experiencia de su saber del absoluto. La facticidad es la propiedad real de toda cosa de ser sin razón y, en consecuencia, "poder sin razón devenir efectivamente otro", 28 mientras que la "factualidad" mienta la no-facticidad de la facticidad: sólo la facticidad no es factual, sólo la contingencia no es contingente. Ciertamente, parece contradictorio hacer de la facticidad, que revelaría la incapacidad del pensamiento para descubrir la razón de lo que es, un absoluto. Pero la peculiaridad de Meillassoux consiste justamente en reponer en la cosa lo que considerábamos una insuficiencia del pensamiento: la ausencia de razón no es un límite del pensamiento en busca de la razón última, sino la propiedad última del ente.

Dicho de otro modo, la irrazón es una propiedad ontológica absoluta, no la marca de la finitud de nuestro saber. Esto es así porque no hay ninguna ley superior que gobierne el devenir, no hay nada más allá o más acá de la "manifiesta gratuidad" de lo dado. El absoluto con el cual nos topamos al

${ }^{25}$ Cfr. Ibidem, pp. 40-42. Watkin analiza esta tesis y examina objeciones de Hallward, Harman y Brassier. Cfr. Watkin, C., Difficult atheism. Post-Theological Thinking in Alain Badiou, Jean-Luc Nancy and Quentin Meillassoux, Edinburgh, EUP, 2011, pp. 132-167.

${ }^{26}$ Cfr. Meillassoux, Q., Après la finitude, chapitre I.

${ }^{27}$ Cfr. Ibidem, pp. 39-40.

28 Meillassoux, Q., “L'Immanence D’Outre Monde”, pp. 41 y 43; también Meillassoux, Q., Après la finitude, pp. 107-108. 
atravesar el círculo correlacional es el Caos al que nada le es imposible -ni siquiera Dios, como veremos más adelante.

2. La segunda tesis enlaza la especulación con una "escatología de la inmortalidad" irreligiosa, en el marco de una crítica vehemente a la posmodernidad fideísta. Detengámonos en la caracterización que hace Meillassoux de nuestra época.

A su juicio, la empresa de des-absolutización crítica de la razón, que le prohíbe el acceso a verdades absolutas, preserva no obstante la posibilidad de una trascendencia impensable, esto es, deja lugar a un residuo de religiosidad y a la defensa de los derechos de la creencia. ${ }^{29}$ El final de la metafísica, "al sustraerle a la razón todas sus aspiraciones al absoluto, tomó la forma de un retorno exacerbado de lo religioso".30 Dicho de otro modo, el correlacionismo moderno no funda ninguna creencia religiosa determinada, pero socava la pretensión de la razón de deslegitimar la creencia por ser irracional: la razón no tiene derecho a discutir la verdad o falsedad del dogma. Des-absolutizar el pensamiento equivale a producir una argumentación fideísta y escéptica contra la pretensión de la razón de acceder a una verdad absoluta capaz de apuntalar o rechazar el valor de la creencia. La clausura contemporánea de la metafísica busca proteger la piedad de toda intrusión de lo racional, lo cual es incompatible con el racionalismo inmanentista de Meillassoux que, como señalamos, propugna un discurso inteligible sobre la esencia última del mundo.

La paradoja de esta situación es que la razón legitima los discursos dirigidos al absoluto a condición de que nada en ellos parezca una justificación racional de su validez: cada uno opone su fe a cada cual y nada de lo que determina nuestras elecciones es demostrable. Los contemporáneos han cedido terreno ante los hombres de fe; si hay una verdad última, queda

${ }^{29}$ En esta línea, por ejemplo, Vattimo sostiene que el fin de la metafísica permite el retorno decisivo de la preocupación religiosa puesto que, una vez clausurada la metafísica, no es posible argumentar que se sabe que Dios no existe. A estas posturas, Meillassoux contrapone las de Lucrecio y Spinoza, que buscan una nueva comprensión racional de la naturaleza de lo divino. Cfr. Meillassoux, Q., "L'Immanence D’Outre Monde”, pp. 45-46.

${ }^{30}$ Meillassoux, Q., Après la finitude, p. 62. 
esperarla de la piedad, no del pensamiento. Meillassoux considera que la Modernidad occidental no fue un vasto movimiento de secularización, sino que el moderno se "en-religó" a medida que se descristianizaba: al mismo tiempo que despojaba al cristianismo de su pretensión de superioridad, se entregó a la "equivalente legitimidad veritativa de todos los cultos". ${ }^{31}$ Frente a este diagnóstico, la propuesta de Meillassoux no es retornar a la fe religiosa sin más pero tampoco sumarse al ateísmo, sino reformular el vínculo entre el pensamiento y la fe, la filosofía y el absoluto. A ello se aboca su perspectiva irreligiosa que presentaremos a continuación.

\section{Dios futuro y Mundo de la Justicia}

1. La tercera tesis, que enlaza "la verdadera inmanencia" con la inmortalidad, quiere resolver el problema del "dilema espectral", que concierne aquellos muertos cuyo duelo no hemos podido hacer por razones esenciales, no meramente psicológicas. Meillassoux afirma que es necesario llevar adelante un "duelo esencial" a fin de lograr una relación viviente, no mórbida, con las muertes terribles y así poder vivir con los espectros esenciales, no morir con ellos. Los "espectros esenciales" son las muertes precoces y odiosas de niños o de padres que saben que sus niños están destinados a lo mismo; muertes terribles, naturales o criminales, que no pueden ser asumidas ni por quienes la sufrieron ni por quienes los sobrevivieron. ${ }^{32}$

Ahora bien, Meillassoux busca superar la alternativa clásica en torno al problema de la relación con los muertos: la respuesta religiosa, que afirma que Dios existe y hay, entonces, un principio de clemencia, trascendente a la humanidad, portador de justicia para los difuntos, y la respuesta atea, que niega que Dios y tal principio existan. La resolución "irreligiosa" quiere compatibilizar la posible resurrección de los muertos -que es la condición religiosa- y la inexistencia de Dios -que es la condición atea. Su postura se sintetiza en el ya célebre enunciado "Dios no existe todavía" [Dieu n'existe pas

\footnotetext{
31 Ibidem, p. 66.

32 Cfr. Meillassoux, Q., “L'Immanence D’Outre Monde”, p. 46 ; Meillassoux, Q., "Dueil à venir, dieu à venir", pp. 1-5.
} 
encore], que expresa la tesis de la "inexistencia divina": existe en la realidad presente la posibilidad de "un Dios futuro", de quien se espera que tenga la potencia de acordar a los espectros algo diferente que su muerte. ${ }^{33}$

En efecto, Dios implica "el advenimiento de un régimen de existencia que me permite esperar para los muertos otra cosa que la muerte". ${ }^{34}$ Meillassoux critica el carácter necesario que tanto la postura religiosa como la atea adjudican a Dios (sea a su existencia o a su inexistencia) y argumenta que la modalidad divina es la de lo posible en un sentido objetivo y futuro, dado que puede realmente producirse en el porvenir; si no hay ley del devenir, el devenir es capaz incluso de Dios. ${ }^{35}$ A diferencia de las potencialidades, que están sometidas a las leyes naturales de nuestro universo y pueden, por tanto, ser probabilizadas, las virtualidades no lo están, por lo que no cabe preguntarse por la probabilidad de que surja una virtualidad como Dios. ${ }^{36}$ Dicho de otro modo, la inexistencia de Dios significa no sólo que no existe (actualmente) sino que, de acuerdo con el principio de factualidad antes explicado, la posibilidad de su existencia es necesaria.

2. Como venimos de puntualizar, la idea de que Dios no existe "aún" o "todavía" [pas encore] significa que actualmente no existe en absoluto, pero esto no tiene por qué durar siempre; se trata de un acontecimiento "realmente posible, eternamente contingente, por siempre incontrolable [immaîtrisable], y completamente improbabilizable". ${ }^{37}$ Se espera del Súper-caos, entonces, que advenga este posible que, quizá, no se produzca jamás, pero del cual es imposible decir que no ocurrirá. Dios es una virtualidad o un surgimiento ex nibilo, un hecho irreductible tal como lo son la materia, irreductible a las teorías físico-matemáticas; la vida, entendida como el conjunto de afecciones, sensaciones y percepciones cualitativas no reductibles a los procesos materiales; y el pensamiento, comprendido como la capacidad de acceder a contenidos inteligibles portadores de verdades eternas. Estos son tres

${ }^{33}$ Meillassoux, Q., “L’Immanence D’Outre Monde”, p. 53.

34 Ibidem, p. 54. Meillassoux lo plantea como "este dios futuro e inmanente..." en Meillassoux, Q., "Dueil à venir, dieu à venir", p. 12.

${ }^{35}$ Véase Watkin, C., Difficult atheism. Post-Theological Thinking in Alain Badiou, Jean-Luc Nancy and Quentin Meillassoux, p. 132-167.

${ }^{36}$ Cfr. Harman, G., Quentin Meillassoux. Philosophy in the making, p. 162.

${ }^{37}$ Meillassoux, Q., “L'Immanence D’Outre Monde”, p. 55. 
mundos, actualmente co-existentes, que se sucedieron en el tiempo, a los cuales Meillassoux agrega el cuarto mundo o mundo de la Justicia, planteado como un surgimiento posible, y que es "el único que podría introducir en el devenir una irreductibilidad y una novedad tan radical como la de la vida con relación a la materia, o del pensamiento con relación a la vida". ${ }^{38}$ Este Mundo del Recomienzo del ser establecería la inmortalidad como garante de la igualdad universal y permitiría "la aspiración más profunda de la ética", que es "la realización universal de la Justicia". 39

Si, como sostiene Harman, la justicia es el verdadero tema de la tesis doctoral de Meillassoux, será preciso aguardar a su publicación (actualizada y ampliada) para presentar un desarrollo cabal del tema. No obstante, podemos adelantar que, para Meillassoux, la injusticia más extrema de todas es la muerte; los seres humanos debemos renacer en condiciones de justicia que superen las muertes horribles de nuestros congéneres. Esta concepción de la justicia constituye, sin dudas, una extravagancia para nuestro mundo presente; ella no sólo se refiere a los vivientes, sino que reclama nuestro rechazo a la injusticia para los muertos -para todos los muertos, dado que es una justicia universal, esto es: sin excepción. Vale subrayar que este mundo de la Justicia debe ser esperado: sólo es posible bajo la condición de que sea deseado en el mundo actual mientras que, si nos limitamos a aguardarlo pasivamente, se vuelve imposible; antes bien, debemos esperarlo activamente, mediante actos de justicia que desplieguen el fervor de una creencia en la universalidad. ${ }^{40}$

Así pues, el proyecto de Meillassoux consiste en "hacer del cuarto Mundo un posible susceptible de suplementar, en nuestro propio Mundo, la subjetividad de los hombres actuales, transformando en profundidad la vida íntima de aquellos que tomen en serio esta hipótesis". ${ }^{41}$ Este cuarto Mundo

${ }^{38}$ Ibidem, p. 56. En este artículo, Meillassoux se refiere de modo indistinto al Cuarto Mundo y al Mundo de la Justicia. Sin embargo, en su tesis doctoral los diferencia. Véase la observación de Harman en Harman, G., Quentin Meillassoux. Philosophy in the making, p. 111.

${ }^{39}$ Meillassoux, Q., “L’Immanence D’Outre Monde”, p. 57.

${ }^{40}$ Cfr. Harman, G., Quentin Meillassoux. Philosophy in the making, pp. 100 y 110-111.

${ }^{41}$ Meillassoux, Q., “L'Immanence D’Outre Monde”, p. 57. 
es un Puede-Ser [Pent-être $]^{42}$ que busca tener efectos en el sujeto escatológico o "escaológico" del presente, aquel que es movido por el deseo de una Justicia universal y una emancipación futura. Se trata de explorar las transformaciones de la subjetividad que resultan de la adhesión a un posible, de liberar la potencia de acción presente del "militante universalista", quien orienta su acción hacia un fin superior posible que puede no advenir nunca. Meillassoux cree que con esta idea se puede quebrar la desesperanza causada por la separación de la justicia y del ser que ocurre tanto en el ateísmo como en la trascendencia religiosa y dar lugar a "una esperanza íntima y vital: un factor de transformación efectivo y emancipador de la subjetividad". ${ }^{43}$ El Mundo de la justicia permitiría emancipar al sujeto por partida doble: de la desesperación, que considera imposible la llegada de una justicia universal para los vivos y los muertos, y del nihilismo, que considera que dicha justicia es pensable y posible aunque no deseable. En suma, el argumento de Meillassoux acerca del Recomienzo hipotético apunta a generar un tipo de sujeto inédito hasta el momento mediante el recurso a una singular forma de esperanza: "creer en Dios porque no existe". ${ }^{44}$

\section{Eterno retorno e inmanencia de otro mundo}

1. La ética de la inmortalidad descansa, como venimos de exponer, en la creencia en un Dios futuro que implica el advenimiento del Mundo de la Justicia. Veamos ahora cómo se vincula con el Eterno Retorno y la inmanencia.

Meillassoux critica la lectura deleuziana del Eterno Retorno y, en general, las interpretaciones "sutiles" del mismo, y defiende "las más simples" pues considera que "la interpretación por lejos más interesante del Eterno Retorno es $[\ldots]$ la que le da su sentido más inmediato y tosco en apariencia”,

\footnotetext{
42 Según el autor, el asunto más importante de la filosofía no es el "ser" sino el "puedeser", en tanto conjuga el principio ontológico de factualidad y el cumplimiento ético de la Justicia universal. Cfr. Ibidem, p. 57.

43 Ibidem, p. 58.

44 Véase Watkin, C., Difficult atheism. Post-Theological Thinking in Alain Badiou, Jean-Luc Nancy and Quentin Meillassoux, p. 148 y ss.
} 
a saber: que todas las cosas vuelven eternamente, bajo la misma forma. ${ }^{45} \mathrm{La}$ hipótesis nietzscheana le interesa a Meillassoux por dos motivos: primero, porque demostraría que no es posible transformar una subjetividad sin una proposición especulativa sobre el mundo. El segundo motivo concierne el concepto de inmanencia, sobre el cual Meillassoux sostiene que "no es de este mundo" [n'est pas de ce monde]:

Creo que con el Eterno Retorno, Nietzsche nos devela una formidable paradoja de la inmanencia [...]: la inmanencia no es de este mundo. Esta tesis, que infiero de Nietzsche, puede oponerse a los diversos pensamientos contemporáneos de la inmanencia, y esencialmente al de Deleuze: a estos pensamientos, es necesario contestar: "nosotros queremos la inmanencia, pero la inmanencia, como Nietzsche comprendió bien, no es de este mundo". ${ }^{46}$

Ahora bien, ¿qué significa que el Eterno Retorno plantea una inmanencia de otro mundo? ¿Cómo entender este oxímoron? Ciertamente, la expresión la inmanencia "de otro mundo" tiene un afán provocativo que Meillassoux explicita en su voluntad de polemizar con Deleuze, no obstante lo cual sería erróneo reducirla a mera retórica.

La interpretación de Meillassoux es que la prueba del Eterno Retorno radica en "asumir una existencia en la cual la muerte no es una interrupción definitiva de nuestra existencia, sino una etapa de nuestro devenir anulada por nuestro renacimiento posterior". ${ }^{47}$ En lugar de ser una prueba destinada a que asumamos nuestro ser-finito y nuestra muerte irremediable, en el Eterno Retorno la muerte es anulada por el retorno de la vida. Así, el desafío que nos impone es el de la inmortalidad, el de la vida sin ningún otro lugar -ni la trascendencia del creyente, ni la nada del ateo-, la vida cerrada sobre su propia potencia ilimitada, la repetición implacable de la existencia. Tal es la paradoja de la inmanencia -la vida deseada al infinito, ilimitada, inmortal-, que es de otro mundo - pues la inmortalidad puede surgir de la mano del advenimiento divino, pero actualmente no existe.

\footnotetext{
45 Meillassoux, Q., “L'Immanence D’Outre Monde”, p. 60.

46 Ibidem, pp. 61-62.

${ }^{47}$ Ibidem, p. 62.
} 
2. Retomemos el argumento de Meillassoux. Los grandes filósofos de la inmanencia como Spinoza y Nietzsche, sostiene, no propusieron pensamientos de la finitud sino de la inmortalidad, comprendida como la perpetuación sin fin de la vida presente (o de una de sus partes). Desde la óptica de Meillassoux, es la finitud la que nos conduce a la trascendencia: la perspectiva de nuestra vida biológica aquí-ahora nos lleva a desear un más allá, mientras que la verdadera aceptación del aquí-ahora consistiría en soportar su prolongación al infinito sin la esperanza de salir a un más allá o a la nada. La auténtica prueba de la inmanencia radical no puede ser efectuada en nuestro mundo inmediato, limitado por la mortalidad biológica, sino que exige pensar un devenir en el cual la vida se abra a sí misma, sin la posibilidad de una salida a otro lugar inconmensurable. Así se enlazan ontología, irreligión y ética: la ontología inmanente, basada en el principio an-hipotético de factualidad, es el sostén de una ética inmanente que afirma esta vida como la única deseable; a diferencia de la religión, no propone otra vida, sino un deseo por esta vida tan poderoso que quiera que ésta sea inmortal; en lugar de prometer la inmortalidad en el paraíso, quiere que nuestra vida presente continúe para siempre, lo cual sólo es posible si adviene Dios. ${ }^{48}$

A la luz de esta interpretación del Eterno Retorno, Meillassoux argumenta que su filosofía de la contingencia eterna permite construir una prueba semejante a la de Nietzsche: la idea de un Recomienzo especulativo, el cuarto Mundo o Mundo de la Justicia. La función sería la misma: hacer la prueba fundamental de la existencia, seleccionar. No obstante, a diferencia del ciclo eterno del retorno de lo mismo, el Recomienzo de los cuerpos se sitúa en una linealidad indefinida y, además, quiere transformar la existencia presente recurriendo a una hipótesis absoluta (el cuarto Mundo), no a una verdad necesaria (el Eterno Retorno). Cabe destacar que la prueba del Recomienzo funciona sólo para quienes buscan la Justicia última y la Igualdad universal, para quienes son sofocados por el duelo de los muertos terribles. El horizonte último al que apunta Meillassoux es el del "comunismo"

${ }^{48}$ Cfr. Harman, G., Quentin Meillassoux. Pbilosophy in the making, p. 97. Hägglund sostiene que el deseo por la inmortalidad está motivado por un deseo por la supervivencia mortal que lo precede y contradice. Cfr. Hägglund, Martin, "Radical atheist materialism: a critique of Meillassoux", en Bryant, L., Srnicek, N. y Harman, G. (eds.), The Speculative Turn. Continental materialism and realism, p. 116. 
comprendido como el fin de la política, esto es, de las relaciones de fuerza, de la astucia, de la guerra, de los sacrificios sangrientos. ${ }^{49}$

\section{Deleuze versus Meillassoux: creer en este mundo}

1. De acuerdo con nuestra hipótesis, cotejar la creencia en el Mundo de la Justicia propuesta por Meillassoux y la "creencia en este mundo" [croyance en ce monde-ci] presentada por Deleuze permitirá poner de relieve afinidades y distancias alrededor de sus concepciones de la inmanencia, la filosofía y la divinidad. Repasemos en qué consiste la idea deleuziana. ${ }^{50}$

En el marco de su descripción del cine moderno, Deleuze sostiene que la ruptura del vínculo que unía al hombre con el mundo conduce al fenómeno de la increencia: "El hecho moderno es que ya no creemos en este mundo. $\mathrm{Ni}$ siquiera creemos en los acontecimientos que nos suceden, el amor, la muerte, como si sólo nos concernieran a medias". ${ }^{11}$ Frente a esta condición de nuestra época, asevera que la salida consiste en "creer, no en otro mundo, sino en el lazo del hombre y del mundo, en el amor o en la vida", 52 y convoca a la invención de "una ética o una fe" que no se dirige a otro mundo ni a un

49 Cfr. Bell, J., "Immanence", en Gratton, P. y Ennis, J. (eds.), The Meillassoux Dictionary, pp. 95-98. Hägglund afirma que Meillassoux despolitiza el trabajo del duelo en la medida en que "desvía la atención de las maneras en que el problema del duelo es mediado históricamente, en favor de una "resolución” general por intervención divina". Cfr. Hägglund, M., "Radical atheist materialism: a critique of Meillassoux", p. 128.

${ }^{50}$ Hemos tratado esta propuesta en Antonelli, M., "Pensar y creer en Derrida y en Deleuze”, en Candioti, M. E., Klocker, D. y Cattaneo, R. (comp.), Perspectivas filosóficas. Investigaciones y contribuciones, Santa Fe, Ediciones UNL, 2009, pp. 265-271; Antonelli, M., "Pérdida del mundo y conversión empirista", en Cabanchik, S. (comp.), Lenguaje, poder y vida. Intervenciones filosóficas, Buenos Aires, Grama Ediciones, 2010, pp. 147-172; y Antonelli, M., "Del resentimiento a la creencia en este mundo. El problema del nihilismo en la obra de Deleuze", Instantes y azares. Perspectivas nietzscheanas, 12, 2014, pp. 169-182.

51 Deleuze, G., Cinéma 2. L'Image-temps, Paris, Minuit, 1985, p. 223. Véase también Deleuze, G., Cinéma 1. L'Image-mouvement, 1983, Paris, Minuit, pp. 279-280.

52 Deleuze, G., Cinéma 2. L'Image-temps, p. 221. 
mundo transformado, sino a este mundo y a esta vida. En una entrevista con Toni Negri deja entrever la dimensión política de su posición: "Creer en este mundo es lo que más falta nos hace; hemos perdido el mundo, hemos sido desposeídos de él. Creer en el mundo es suscitar acontecimientos incluso pequeños que escapen al control, o hacer nacer nuevos espacios-tiempo, aun de superficie o de volumen reducido". 53

En Qu'est-ce que la philosophie?, la creencia recibe el nombre de "conversión empirista", y designa la confianza en las posibilidades que ofrece el mundo para que nazcan nuevos modos de existencia. ${ }^{54}$ Deleuze se desplaza desde la creencia en cuanto tal al modo de existencia de aquel que cree y acude al célebre argumento de Pascal para ilustrar esta perspectiva: aun si parece versar sobre la existencia trascendente de Dios, "lo que está en juego en la apuesta, aquello sobre lo cual se apuesta, es la existencia inmanente de aquél que cree que Dios existe". ${ }^{55}$ Dicho de otro modo, el caballero de la fe de Kierkegaard y el apostador pascaliano no se preocupan, en verdad, por la existencia trascendente de Dios, sino por "las posibilidades inmanentes infinitas que aporta la existencia de aquél que cree que Dios existe". ${ }^{56}$

Según Deleuze, "nuestro problema" ya no concierne la existencia o la inexistencia de Dios, sino que consiste en cómo volver a creer en este mundo. El plano de inmanencia contemporáneo se distingue del anterior porque nuestro problema ya no gira en torno a Dios:

Es posible que creer en este mundo, en esta vida, haya devenido nuestra tarea más difícil, o la tarea de un modo de existencia por descubrir sobre nuestro plano de inmanencia hoy. Es la conversión empirista (tenemos tantas razones para no creer en el mundo de los hombres, hemos perdido

53 Deleuze, G., "Contrôle et devenir", en Pourparlers, Paris, Minuit, 2005, p. 239.

${ }^{54}$ Cfr. Deleuze, G. y Guattari, F., Qu'est-ce que la philosophie?, Paris, Minuit, 1991, pp. $72-73$.

55 Ibidem, p. 71. Véase Deleuze, G., Cinéma 1. L'Image-mouvement, pp. 160-161.

56 Deleuze, G. y Guattari, F., Qu'est-ce que la philosophie?, p. 72. En L'image-temps se distinguen otros modos de existencia, además del creyente y el no creyente: el devoto, el escéptico, el hombre del mal. Cfr. Deleuze, G., Cinéma 2. L'Image-temps, pp. 230231. 
el mundo, peor que una novia, un hijo o un dios...). Sí, el problema ha cambiado. ${ }^{57}$

Cabe destacar que Deleuze emplea la idea de "conversión" en el sentido opuesto al de adherir a una religión dado que se trata de una "conversión atea", una creencia "que está lista para pasar del lado del ateo". En efecto, la creencia que apunta a este mundo surge de una mutación que la vuelve laica, mundana, inmanente. ${ }^{58}$ Nuestro autor encuentra esta perspectiva en el empirismo humeano, definido como una empresa que naturalizó o "laicizó" la creencia..$^{59} \mathrm{El}$ viraje operado por Hume en filosofía consiste en establecer la creencia como base del conocimiento ("todo es creencia"). ${ }^{60} \mathrm{En}$ este sentido, la creencia en este mundo radicaliza el movimiento del pensamiento moderno, en tanto ella "sólo remplaza al saber cuando se hace creencia en este mundo, tal como es". ${ }^{61}$

${ }^{57}$ Deleuze, G. y Guattari, F., Qu'est-ce que la philosophie?, pp. 72-73. La creencia constituye una disposición a la acción, esto es: provoca una determinada actitud en el sujeto tendiente a religarlo al mundo. Véase M. Lazzarato, "Del conocimiento a la creencia, de la crítica a la producción de subjetividad", trad. de Marcelo Expósito, 2008. Disponible en http://eipcp.net/transversal/0808/lazzarato/es/print ${ }^{58}$ Cfr. Deleuze, G., Cinéma 2. L’Image-temps, p. 230.

${ }^{59}$ Deleuze esboza la idea de una "comunidad de hermanos" o "sociedad sin padres" que remplacen el conocimiento por la confianza: "hace falta una comunidad nueva, cuyos miembros sean capaces de "confianza", es decir, de esa creencia en sí mismos, en el mundo, en el devenir”. Cfr. Deleuze, G., Critique et clinique, Paris, Minuit, 2002, pp. 111-112.

${ }^{60}$ Deleuze, G. (2004), L'T̂le déserte et autres textes. Textes et entretiens 1953-1974, edición de D. Lapoujade, Paris, Minuit, p. 232 y ss.; cfr. Deleuze, G., Deux régimes de fous. Textes et entretiens 1975-1995, edición de D. Lapoujade, Paris, Minuit, 2003, p. 326. En L'image-temps, el "giro decisivo de la filosofía" (remplazar el modelo del saber por el de la creencia) no sólo remite a Hume, sino que va "de Pascal a Nietzsche". Cfr. Deleuze, G., Cinéma 2. L'Image-temps, p. 224.

${ }^{61}$ Ibidem, p. 224. En Qu'est-ce que la philosophie? se distingue una imagen "clásica" del pensamiento, orientada hacia lo verdadero, y una imagen "moderna", de raíz humeana, que renuncia al vínculo con la verdad y sustituye el conocimiento por la creencia. En dicho contexto, la creencia deviene un movimiento del pensamiento independiente de la religión. Cfr. Deleuze, G. y Guattari, F., Qu'est-ce que la philosophie?, pp. 54-55. 
2. Ensayemos una confrontación entre las propuestas de Deleuze y Meillassoux. En primer lugar, ambas coinciden en destacar los efectos subjetivos de la creencia: el modo en que afecta al militante universalista de Meillassoux, o bien al creyente o al apostador de Deleuze. Como hemos señalado, Deleuze insiste en que lo que está en juego en la apuesta de Pascal y en la elección de Kierkegaard es el modo de existencia de aquel que apuesta o elige. Meillassoux, por su parte, subraya que su planteo busca explorar cómo se transforma una subjetividad si cree en el advenimiento del mundo de la justicia: el sujeto logra hacer el duelo esencial y evitar la desesperación y el nihilismo. También es posible constatar la relevancia otorgada al afecto del sujeto transformado: Meillassoux alude al "ardor" [ardeur] de la orientación emancipadora, el "afecto que nos domina y por el cual somos los herederos de todos los movimientos emancipadores y escatológicos del pasado"; 62 Deleuze, por su parte, realza el entusiasmo inmanente como el verdadero triunfo de una revolución. ${ }^{63}$

No obstante, sería desacertado quitarle toda relevancia al objeto en que convoca a creer Meillassoux: en su planteo hay una fuerte afirmación ontológica sobre la cual se erige la reflexión ética. El Puede-ser o Súper-caos es la base de la creencia en la posibilidad de que Dios advenga; la inexistencia divina en el presente da lugar la existencia virtual de un Dios futuro. Deleuze, por el contrario, relativiza aquello en lo que se cree, apuesta o elige, y repite que lo que está en juego es la manera de ser de aquél que cree, apuesta o elige: se trata de una conversión inmanentista de la fe cuyo único objeto son los modos de existencia. ${ }^{64}$ Además, Deleuze llama a creer en algo que existe -el mundo, esta vida, nuestro lazo ahora quebrado con él-, mientras que Meillassoux reclama una creencia en algo que no existe (todavía). De allí que la proposición especulativa corresponda a Meillassoux antes que a Deleuze,

\footnotetext{
${ }^{62}$ Meillassoux, Q., “L’Immanence D’Outre Monde”, p. 65.

${ }^{63}$ Cfr. Deleuze, G. y Guattari, F., Qu'est-ce que la philosophie?, pp. 96-97 y 167.

${ }^{64}$ Cfr. Zabunyan, D., Gilles Delenze. Voir, parler, penser an risque du cinema, Paris, PU Sorbonne Nouvelle, 2006, p. 194.
} 
quien más bien proclama una proposición empirista que llama a creer en el cuerpo. ${ }^{65}$

En segundo lugar, ambos convocan primariamente a creer y asumen que es a partir de la creencia que el sujeto actuará. Meillassoux argumenta que, al igual que en el Eterno Retorno nietzscheano, su proposición especulativa persigue efectos prácticos, mientras que Deleuze no explicita por qué es necesario plantear una relación de creencia para reinventar el lazo con el mundo. Por otra parte, Meillassoux no desarrolla puntualmente el concepto de creencia, al que se refiere en términos de "esperanza", la "forma inmanente de la esperanza". ${ }^{66}$ Deleuze tampoco se detiene en la noción, pero multiplica los sinónimos: confianza, conversión empirista, ética, fe. Vale observar que los dos autores coinciden en la referencia al argumento de la apuesta de Pascal. ${ }^{67}$

En tercer lugar, el Mundo de la Justicia perfilado por Meillassoux constituye, desde una perspectiva deleuziana, una visión moral, no ética, del mundo. Deleuze elabora una ética que defiende los criterios de valoración inmanentes a la vida en la línea de Spinoza y Nietzsche, que son "autores de la inmanencia" porque rechazan toda trascendencia, esto es, "toda instancia que supere a la Tierra y a los seres humanos". ${ }^{68} \mathrm{Si}$ bien el Dios de Meillassoux no es creador, sí es moral en la medida en que se apoya, implícitamente, en la oposición universal Bien-Mal, mientras que la ética deleuziana se despliega en

${ }^{65}$ Creer "es simplemente creer en el cuerpo" (Deleuze, G., Cinéma 2. L'Image-temps, p. 225). Véase Ibidem, p. 245 y ss.; Zourabichvili, F., Deleuze, une philosophie de l'événement, Paris, PUF, 1994, pp. 69-70.

${ }^{66}$ Cfr. Meillassoux, Quentin, L'Inexistence divine, p. 389.

${ }^{67}$ Así lo plantea Meillassoux en el último capítulo de su tesis doctoral: "Le nouveau pari", que expone "una apuesta filosófica que sucede a la apuesta religiosa de Pascal". Cfr. Ibidem, pp. 373-376.

68 Deleuze, G. y Parnet, C., L'Abécédaire de Gilles Delenze. Paris, Vidéo Éd. Montparnasse, 1996, "H" comme Histoire de la philosophie. Hemos desplegado la contraposición entre la ética basada en la inmanencia y la moral en la trascendencia en Antonelli, M., "El concepto de inmanencia práctica en Deleuze", Ideas y V alores, Universidad Nacional de Colombia, LXVI, 164, 2017, pp. 317-341. 
maneras de ser buenas o malas no hipostasiadas (lo bueno y lo malo, lo noble y lo vil, lo alto y lo bajo).

Como observa Gratton, Meillassoux se sitúa lejos de la afirmación de la vida propia de inmanentismos como los de Spinoza y Deleuze: para él, nuestra vida tal como es ahora se ve confrontada con el absurdo de Camus; el posible advenimiento ex nibilo de la Justicia es la única esperanza para redimir este mundo injusto. ${ }^{69}$ Esta vida (de injusticias, finitud, diferencias, política) es afirmada sólo en la medida en que desaparecerá en beneficio de otra, la inmortal, que parece ser el verdadero objeto de la afirmación; en otras palabras, afirmaremos aquella vida inmortal, no esta vida del mundo de la finitud $-\mathrm{O}$ bien ésta, pero sólo en la medida en que deje paso a aquella. ${ }^{70}$ Además, la idea de Meillassoux acerca de la inmortalidad aproxima su planteo al Sistema del Juicio que Deleuze describió como una doctrina moral y teológica, referida al Apocalipsis cristiano, que se erige no sólo sobre una deuda infinita e impagable con la divinidad sino sobre el carácter inmortal de la existencia. ${ }^{71}$

En cuarto lugar, el Mundo de la Justicia puede ser considerado un Ideal suprasensible o incluso un horizonte regulador en la medida en que introduce una trascendencia que remite la vida y sus potencias a una normatividad superior, a valores que la regulan. ${ }^{72}$ Deleuze considera que luchar contra la moral implica rechazar valores superiores a la vida, como es la Justicia para Meillassoux. Si bien, por razones de espacio y de pertinencia temática, no podemos abordar aquí el lazo entre el nihilismo y la trascendencia, cabe observar que, según la crítica nietzscheana prolongada por Deleuze, los ideales de cualquier índole envuelven una posición nihilista en la medida en que desdoblan la realidad introduciendo un corte o una discontinuidad entre la realidad real y la ideal. Desde esta perspectiva, Meillassoux apela a una peculiar interpretación del Eterno Retorno nietzscheano en un horizonte alejado de Nietzsche, en tanto se encuentra asediado por la trascendencia y el

\footnotetext{
${ }^{69}$ Cfr. Gratton, P., Speculative realism. Problems and prospects, p. 69.

${ }^{70}$ Cfr. Ibidem, p. 84.

${ }^{71}$ Cfr. Deleuze, G., Critique et clinique, p. 158.

${ }^{72}$ Cfr. Antonelli, M., "El problema de la utopía en Gilles Deleuze”, Isegoría, CSIC, 47, 2012, pp. 519-539; Mengue, P., Utopies et devenirs deleuziens, Paris, L'Harmattan, 2009, p. 23 y ss.
} 
nihilismo -aun si, desde la visión de Meillassoux, su propuesta permitiría esquivar el nihilismo. De acuerdo con Deleuze, no puede tener lugar una inmanencia "de otro mundo" pues el "otro mundo" (sea un más allá inmortal, sea un mundo distinto o transformado) implica una trascendencia; de allí que enfatice que no se trata de creer en otro mundo sino en este mundo [ce mondeci].

En quinto lugar, las divergencias entre ambos autores se patentizan en el lazo entre la filosofía y la religión. Deleuze asocia la filosofía con la inmanencia y la opone a la religión y lo divino: "Hay religión cada vez que hay trascendencia, Ser vertical, Estado imperial en el cielo o sobre la tierra, y hay filosofía cada vez que hay inmanencia". ${ }^{73}$ Ciertamente, Meillassoux podría objetar que su proyecto no corresponde a una religión sino a una "irreligión" en la medida en que Dios no existe (todavía) y que el retorno por el que aboga no es el de la religión sino el del mesianismo: a su juicio, lo que está en juego en Occidente es cómo pensar la unidad de la religión judía y de la razón griega, el mesianismo igualitario de los judíos y la racionalidad inmanente de los griegos. ${ }^{74}$ Desde esta óptica, la opinión de Johnston acerca de que Meillassoux "contrabandea [smuggles] idealidad religiosa" mediante su materialismo no constituiría una auténtica objeción a su planteo. ${ }^{75}$

Para Meillassoux "la irreligión filosófica no es un ateísmo sino la condición de un verdadero acceso a lo divino"; 76 la Filosofía no rechaza a Dios sino que debe hablar de Dios, que es "lo que está en juego en la disputa entre inmanencia y trascendencia". ${ }^{77}$ Por el contrario, Deleuze adjudica a la filosofía un ateísmo intrínseco, como queda claro cuando atribuye a François Châtelet el establecimiento de un "puro campo de inmanencia" mediante un "ateísmo tranquilo" que consiste en "una filosofía para la cual Dios no es un problema, la inexistencia o incluso la muerte de Dios no son problemas, sino al contrario las condiciones que es necesario considerar adquiridas para hacer surgir los

${ }^{73}$ Cfr. Deleuze, G. y Guattari, F., Qu'est-ce que la philosophie?, pp. 45-46.

74 Meillassoux, Q., L'Inexistence divine, "Conclusion".

75 Cfr. Johnston, Adrian, “Hume's revenge: À Dieu, Meillassoux?”, Bryant, L., Srnicek, N. y Harman, G. (eds.), The Speculative Turn. Continental materialism and realism, p. 113.

76 Meillassoux, Q., “L'Immanence D’Outre Monde”, p. 39.

77 Watkin, C., Difficult atheism, p. 134. 
verdaderos problemas". ${ }^{78}$ Esta posición se emparenta con la ya mencionada a propósito de la conversión empirista: en nuestro plano de inmanencia, Dios ya no el problema fundamental; la creencia en Dios ha dejado paso a la creencia en el mundo como objeto de la apuesta o de la elección.

Por su parte, el ateísmo que propugna Deleuze puede ser considerado, desde la óptica de Meillassoux, como una ratificación de la demarcación religiosa entre la trascendencia y la inmanencia. A su juicio, el ateo está "satisfecho" con el "territorio insatisfactorio" que la religión le cede; se comienza admitiendo que el territorio de la inmanencia es como la religión lo describe, luego se declara dicho territorio como el único que existe y, finalmente, se inventan modos de volverlo vivible a pesar de ese hecho. De acuerdo con esta perspectiva, el ateo y el hombre de la religión comparten "el carácter trágico de la inmanencia". ${ }^{79}$

Por último, a propósito de la tesis de Meillassoux que anuda la inmanencia con la inmortalidad y la trascendencia con la finitud, es necesario observar que también Deleuze ha señalado un más allá de la finitud y la muerte del hombre, pero no ligado a la inmortalidad. En el Apéndice a Foucault, desarrolla la hipótesis según la cual la finitud es la marca de la formación histórica del siglo XIX, dado que las fuerzas en el hombre entraron en relación con las fuerzas del afuera (la vida, el trabajo, el lenguaje) y dieron lugar a la forma-Hombre.$^{80}$ De este modo, la finitud constituyente reemplazó al infinito originario de la formación histórica clásica, que ponía en comunicación las fuerzas en el hombre con las fuerzas de elevación al infinito, lo cual redundaba en la forma-Dios. Deleuze detecta signos de una mutación en las relaciones de fuerzas, que darán lugar a una forma aún desconocida. Las fuerzas en juego (el silicio, los componentes genéticos, las expresiones a-gramaticales) ya no son las del infinito ni las de la finitud, sino las de un finito-ilimitado, que designa situaciones en las cuales un número finito de componentes ofrece una diversidad casi ilimitada de combinaciones. En suma, Deleuze se desplaza hacia un horizonte post-finito, pero no lo hace desde la especulación, como Meillassoux, sino situando las distintas formas en la historia y analizando las

${ }^{78}$ Deleuze, G., Périclès et Verdi. La philosophie de François Châtelet, Paris, Minuit, 1996, p. 7, cursivas nuestras.

${ }^{79}$ Harman, G., Quentin Meillassoux. Philosophy in the making, p. 117.

${ }^{80}$ Cfr. Deleuze, G., Foucault, Paris, Minuit, 2004, pp. 131-141. 
mutaciones en las relaciones de fuerzas. Así, el más-allá-de-la-finitud no vendrá por un giro especulativo en filosofía, sino por una mutación histórica que incluye el pensamiento filosófico, pero no depende de él.

\section{Observaciones finales}

Hemos iniciado un contrapunto entre Meillassoux y Deleuze a propósito de la inmanencia que deberá ser continuado y ampliado. Nos focalizamos en la tesis de Meillassoux que reúne la inmanencia del Súper-caos y la creencia en el Mundo de la justicia, la inmortalidad que deriva del Eterno Retorno y el Dios virtual. Hemos contrastado esta paradójica ética de la creencia que se proclama inmanente pero apunta a la resurrección de los cuerpos, con la creencia en este mundo y la solidaridad entre filosofía y ateísmo postulada por Deleuze.

Mediante la idea de una inmanencia de otro mundo, Meillassoux quiere diferenciar su inmanentismo del de Deleuze puesto que éste ha elaborado un pensamiento reconocido en el campo filosófico francés contemporáneo, reinventando un concepto de antiguo origen en nuestra tradición filosófica. Hemos ensayado "respuestas" que Deleuze podría ofrecer a la crítica de Meillassoux, así como contra-argumentos de éste, con vistas a enriquecer el debate. Desde una perspectiva deleuziana, pese a que Meillassoux concibe su filosofía como una filosofía de la inmanencia que evitaría las insuficiencias del ateísmo y la finitud, reintroduce motivos que Deleuze asocia ineludiblemente a la trascendencia: la inmortalidad y lo divino. El recurso a la inmanencia del más allá no haría sino devolver una trascendencia encubierta, disimulada o disfrazada. La propuesta de Meillassoux se ajusta, paradójicamente, a la descripción que Deleuze hace de la fenomenología, a la que Meillassoux critica por su correlacionismo: se espera, de la inmanencia, una ruptura hacia la trascendencia; se pretende que la inmanencia (el Súper-caos) "fabrique" la trascendencia (Dios). ${ }^{81}$

Para Deleuze, la inmanencia es de este mundo o se vuelve trascendencia; la filosofía es atea o se convierte en religión; el pensamiento

${ }^{81}$ Cfr. Deleuze, G. y Guattari, F., Qu'est-ce que la philosophie?, pp. 48-49. 
debe abandonar el tema "Dios" para abocarse a los auténticos problemas de nuestra época entre los cuales sobresale el nihilismo, al que es preciso enfrentar mediante una confianza en este mundo, no mediante una fe en otro mundo.

\section{Bibliografía consultada}

Abadi, Diego, "Some possible relations between Quentin Meillassoux's "speculative materialism» and Gilles Deleuze's «transcendental empiricism »", Journées "Les temps des choses, la substance du temps", 21 et 22 mai 2015, Université Paris 8.

Agamben, Giorgio "L'immanence absolue", en Alliez, Eric (dir.), Gilles Deleuze. Une vie philosophique, Paris, Synthélabo, 1998, pp. 165-188.

Antonelli, Marcelo, "Pensar y creer en Derrida y en Deleuze", en Candioti, M. E.; Klocker, D; Cattaneo, R. (comp.), Perspectivas filosóficas. Investigaciones y contribuciones, Santa Fe, Ediciones UNL. 2009. pp. 265 - 271.

Antonelli, Marcelo "Pérdida del mundo y conversión empirista", en Cabanchik, S. (comp.), Lenguaje, poder y vida. Intervenciones filosóficas, Buenos Aires, Grama Ediciones, 2010, pp. 147-172.

Antonelli, Marcelo, "El capitalismo según Gilles Deleuze: inmanencia y fin de la historia", Cuadernos de Filosofía, UBA, 57, 2011, pp. 51-66.

Antonelli, Marcelo, El concepto de inmanencia en la filosofía de Deleure, tesis doctoral, UBA-Paris 8, defendida el 14 de marzo de 2012.

Antonelli, Marcelo, "El problema de la utopía en Gilles Deleuze", Isegoría, CSIC, 47, 2012, pp. 519-539.

Antonelli, Marcelo, "Vitalismo y desubjetivación. La ética de la prudencia en Gilles Deleuze”, Signos Filosóficos, UAM, Unidad Iztapalapa, 15, 30, 2013, pp. 89-117.

Antonelli, Marcelo, "Pensar la inmanencia: Gilles Deleuze y François Jullien", Eidos. Revista de filosofía de la Universidad del Norte, 19, 2013, pp. 12-45. 
Antonelli, Marcelo, "Sobre el origen del concepto de inmanencia en Gilles Deleuze", INTERthesis, Revista Internacional Interdisciplinar, UFSC, 11, 2, 2014, pp. 15-36.

Antonelli, Marcelo, "Del resentimiento a la creencia en este mundo. El problema del nihilismo en la obra de Deleuze", Instantes y azares. Perspectivas nietzscheanas, 12, 2014, pp. 169-182.

Antonelli, Marcelo, "El concepto de inmanencia práctica en Deleuze", Ideas y Valores, Universidad Nacional de Colombia, LXVI, 164, 2017, pp. 317-341.

Badiou, Alain, Deleuze: La clameur de l'être, Paris, Hachette, 1997.

Bell, Jeoffrey, "Immanence", en Gratton, Peter y Ennis, Paul J. (eds.), The Meillassoux Dictionary, Edinburgh, EUP, 2015, pp. 95-98.

Bryant, L., Srnicek, N. y Harman, G., "Towards a Speculative Philosophy", en Bryant, L., Srnicek, N. y Harman, G. (eds.), The Speculative Turn. Continental materialism and realism, Melbourne, Re.press., 2011, pp. 1-18.

Ciccarelli, Roberto, Immanenza. Filosofia, diritto e politica della vita dal XIX al XX secolo, Bologna, Il Mulino, 2008.

Deleuze, Gilles, Deux régimes de fous. Textes et entretiens 1975-1995, edición de D. Lapoujade, Paris, Minuit, 2003.

Deleuze, Gilles, Critique et clinique, Paris, Minuit, 2002.

Deleuze, Gilles, Périclès et Verdi. La philosophie de Francois Châtelet, Paris, Minuit, 1996.

Deleuze, Gilles, "Contrôle et devenir", en Pourparlers, Paris, Minuit, 2005, pp. 229-239.

Deleuze, Gilles, Cinéma 2. L'Image-temps, Paris, Minuit, 1985.

Deleuze, Gilles, Cinéma 1. L'Image-mouvement, Paris, Minuit, 1983.

Deleuze, Gilles y Guattari, Félix, Qu'est-ce que la philosophie?, Paris, Minuit, 1991.

Deleuze, Gilles y Parnet, Claire, L’Abécédaire de Gilles Deleure, Paris, Vidéo Éd. Montparnasse, 1996. 
DeLanda, Manuel, Intensive science and virtual philosophy, London/New York, Bloomsbury, 2002.

DeLanda, Manuel y Harman, Graham, The rise of realism, Cambridge, Polity Press, 2017.

Duru, Martin, "La résurrection qui vient...", Philosophie Magazine, 25, 2012 2013 [http://www.philomag.com/les-idees/la-resurrection-qui-vient-6491].

Gratton, Peter, Speculative realism. Problems and prospects, London/New York, Bloomsbury, 2014.

Gratton, Peter y Ennis, Paul J. (eds.), The Meillassoux Dictionary, Edinburgh, EUP, 2015.

Hägglund, Martin, "Radical atheist materialism: a critique of Meillassoux", en Byrant, L., Srnicek, N. and Harman, G. (eds.), The Speculative Turn. Continental materialism and realism, Melbourne, Re.press., 2011, pp. 114-129.

Harman, Graham, Quentin Meillassoux. Philosophy in the making, Edinburgh, EUP, 2011.

Johnston, Adrian, “Hume's revenge: À Dieu, Meillassoux?” en Bryant, L., Srnicek, N. and Harman, G. (eds.), The Speculative Turn. Continental materialism and realism, Melbourne, Re.press., 2011, pp. 92-113.

Lazzarato, Maurizio, "Del conocimiento a la creencia, de la crítica a la producción de subjetividad”, trad. de Marcelo Expósito, 2008. Disponible en http:/ / eipcp.net/transversal/0808/lazzarato/es/print

Longo, Anna (2014): "The contingent emergence of thought: a comparison between Meillassoux and Deleuze", en Meillassoux, Quentin: Time without becoming, ed. por A. Longo, Mimesis International, pp. 31-50.

Meillassoux, Quentin, "Dueil à venir, dieu à venir", Paris, Ed. Ismael, 2017, reedición del artículo publicado en Critique, 704-705, "Dieu", janvier-février 2006, Paris, Minuit, pp. 105-115. Disponible en https://editions-ismael.com

Meillassoux, Quentin, “ «There is contingent being independent of us, and this contingent being has no reason to be of a subjective nature »: Interview with Quentin Meillassoux", en Van der Tuin, Eric and Dolphjin, Rick (eds.), 
New Materialism: Interviews and Cartographies, Michigan, Open Humanities Press, 2012, pp. 71-81.

Meillassoux, Quentin, "Potentiality and Virtuality", en Bryant, L., Srnicek, N. and Harman, G. (eds.), The Speculative Turn. Continental materialism and realism, Melbourne, Re.press, 2011, pp. 224-236.

Meillassoux, Quentin, “Appendix: Excerpts from L'Inexistence divine”, en Harman, G., Quentin Meillassoux. Philosophy in the making, Edinburgh, EUP, 2011, pp. 175-238.

Meillassoux, Quentin, “L'Immanence D'Outre Monde”, ETHICA, Rio de Janeiro, 16, 2, 2009, pp. 39-71.

Meillassoux, Quentin, "Substraction and contraction: Deleuze, immanence, and Matter and Memory", en Mackay, R. (ed.), Collapse III, Falmouth, Urbanomic, 2007, pp. 63-107.

Meillassoux, Quentin, "Presentation", en Mackay, R. (ed.), Collapse III, Falmouth, Urbanomic, 2007, pp. 408-449.

Meillassoux, Quentin, Après la finitude. Essai sur la nécessité de la contingence, Paris, Ed. Du Seuil, 2006.

Meillassoux, Quentin, L'Inexistence divine, tesis doctoral, Université de Paris I, Département de philosophie, 1997.

Mengue, Philippe, Utopies et devenirs deleuriens, Paris, L'Harmattan, 2009.

Ramírez, Mario Teodoro, "Devenir inmortal. La crítica de Quentin Meillassoux a la filosofía de la inmanencia de Gilles Deleuze", Signos Filosóficos, XVIII, 35, enero-junio 2016, pp. 32-51.

Watkin, Christopher, Difficult atheism. Post-Theological Thinking in Alain Badiou, Jean-Luc Nancy and Quentin Meillassoux, Edinburgh, EUP, 2011.

Zabunyan, Dork, Gilles Deleuze. Voir, parler, penser au risque du cinéma, Paris, PU Sorbonne Nouvelle, 2006.

Zizek, Slavoj y Woodard, Ben, "Interview", en Bryant, L., Srnicek, N. y Harman, G. (eds.), The Speculative Turn. Continental materialism and realism, Melbourne, Re.press, 2011, pp. 406-415. 
Zouravichvili, François, Deleuže, une philosophie de l'événement, Paris, PUF, 1994.

Recibido: 04/2018; aceptado: 02/ 2019. 